\title{
Complicações Neurológicas oriundas da infecção por SARS-CoV-2: uma revisão da literatura
}

\author{
Neurological complications from SARS-CoV-2 infection: \\ a literature review
}

\section{Complicaciones neurológicas de la infección por SARS-CoV- 2: una revisión de la literatura}

\author{
Vinicius Faustino Lima de Oliveira ${ }^{1}$, Elane de Nazaré Magno Ferreira² \\ 1.Discente de Medicina da Universidade Federal do Amapá - UNIFAP. Departamento de \\ Ciências Biológicas e da Saúde - DCBS. Macapá-AP, Brasil. \\ 2.Biomédica (UFPA), Doutora em Neurociências (UNIFESP), Professora de Histologia e \\ Fisiopatologia no curso de Medicina, Departamento de Ciências Biológicas e da Saúde, \\ Universidade Federal do Amapá (UNIFAP), Macapá-AP, Brasil.
}

\begin{abstract}
Resumo
Introdução. A COVID-19 é causada pelo coronavirus 2 da síndrome respiratória aguda grave (SARS-CoV-2), que teve seus primeiros casos relatados em dezembro de 2019. O vírus apresenta material genético contituido de RNA de fita simples. SARS-CoV-2 apresenta capacidade de invasão de tecidos que não estão contidos no sistema respiratório e a invasão do sistema nervoso central vêm sendo documentada na literatura. Objetivos. Apontar os sintomas e complicações neurológicas relatados em pacientes infectados pelo SARS-CoV- 2 . Método. A pesquisa foi realizada nas bases de dados PUBMED, EMBASE e Google Acadêmico sem restrição de idiomas. Houve a combinação das palavras-chave "covid-19", "neurology" e "SARS-CoV-2". Resultados. Febre estava presente em 48,14\% dos pacientes, paresias em $40,74 \%$ e estado mental alterado em $33,33 \%$ dos indivíduos. Distúrbios do olfato e do paladar estão presentes em $14,81 \%$ dos casos. Afasia foi descrita em $7,40 \%$ dos pacientes, disartria em $25,92 \%$, ataxia $14,81 \%$ e hemiplegia em $22,22 \%$ dos casos. Ademais, $48,14 \%$ sofreram algum tipo de acidente vascular cerebral (AVC), enquanto 25,92\% cursaram com Síndrome de Guillain Barré (SGB) e 14,81\% evoluíram com encefalite. Conclusão. Ainda há poucas evidências que permitam afirmar com precisão quais são os mecanismos exatos de invasão do tecido nervoso, mas já se tem a evidência que esse processo existe.
\end{abstract}

Unitermos: COVID-19; SARS-Cov-2; neurologia, Sistema nervoso

\begin{abstract}
Introduction. COVID-19 is caused by coronavirus 2 of severe acute respiratory syndrome (SARS-CoV-2), which had its first cases reported in December, 2019. The virus presents genetic material constituted with single-stranded RNA. SARS-CoV-2 has the ability to invade tissues that are not contained in the respiratory system and the central nervous system invasion has been documented in the literature. Objectives. To point out the neurological symptoms and complications reported in patients infected with SARS-CoV-2. Method. The research was conducted in PUBMED, EMBASE and Google Scholar without language restriction. There was the combination of the keywords "covid-19", "neurology" and "SARS-CoV-2". Results. Fever was present in $48.14 \%$ of patients, paresis in $40.74 \%$ and altered mental status in $33.33 \%$ of individuals. Smell and taste disorders are present in $14.81 \%$ of cases. Aphasia was described in $7.40 \%$ of patients, dysarthria in $25.92 \%$, ataxia $14.81 \%$, and hemiplegia in $22.22 \%$ of cases. In addition, $48.14 \%$ suffered some type of stroke, while $25.92 \%$ had Guillain Barré Syndrome (GBS) and 14.81\% developed encephalitis. Conclusion. There is still few evidence to accurately state the exact mechanisms of nervous tissue invasion, but there is already evidence that this process exists.
\end{abstract}

Keywords: COVID-19; SARS-Cov-2; neurology, nervous system 


\begin{abstract}
Resumen
Introducción. COVID-19 es causada por coronavirus 2 del síndrome respiratorio agudo grave (SARS-CoV-2), que tuvo sus primeros casos notificados en diciembre de 2019. El virus presenta material genético contituado con ARN de una sola cadena. SARS-CoV-2 tiene la capacidad de invadir tejidos que no están contenidos en el sistema respiratorio y la invasión del sistema nervioso central se ha documentado en la literatura. Objetivos. Señalar los síntomas neurológicos y las complicaciones notificadas en pacientes infectados con SARS-CoV2. Método. La investigación se llevó a cabo en pubmed, EMBASE y Google Scholar sin restricciones de idioma. Hubo la combinación de las palabras clave "covid-19", "neurología" y "SARS-CoV-2". Resultados. La fiebre estuvo presente en el $48,14 \%$ de los pacientes, la paresia en el $40,74 \%$ y alterada el estado mental en el $33,33 \%$ de los individuos. Los trastornos del olfato y del gusto están presentes en el $14,81 \%$ de los casos. La afasia se describió en el $7,40 \%$ de los pacientes, la disartria en el $25,92 \%$, la ataxia el $14,81 \%$ y la hemiplejia en el $22,22 \%$ de los casos. Además, el $48,14 \%$ sufrió algún tipo de accidente cerebrovascular, mientras que el $25,92 \%$ padecía el Síndrome de Guillain Barré (SGB) y el $14,81 \%$ desarrollaban encefalitis. Conclusión. Todavía hay poca evidencia para indicar con precisión los mecanismos exactos de invasión de tejido nervioso, pero ya hay evidencia de que este proceso existe.
\end{abstract}

Palabras clave: COVID-19; SARS-Cov-2; neurología, sistema nervioso

Trabalho realizado na Universidade Federal do Amapá (UNIFAP), Macapá-AP, Brasil.

\title{
INTRODUÇÃO
}

A COVID-19 é a doença resultante da infecção pelo coronavírus 2 da síndrome respiratória aguda grave (SARSCoV-2), que teve seus primeiros casos na China em dezembro de 2019¹. O vírus apresenta material genético constituído de uma fita simples de RNA de $\beta$-coronavírus, com alto grau de similaridade com o SARS-CoV 2,3 .

A infecção pelo SARS-CoV-2 não se restringe apenas aos órgãos do sistema respiratório e a capacidade de invasão aos tecidos neurológicos tem sido amplamente descrita na literatura, e é mais frequente em casos graves da doença e em indivíduos idosos ${ }^{4-6}$. Mao et al (2020), em um estudo retrospectivo usando prontuários de pacientes de hospitais 
localizados em Wuhan e que desenvolveram a COVID-19, relatam que cerca de $36 \%$ dos pacientes infectados cursam com manifestações relacionadas ao sistema nervoso central $(\mathrm{SNC})^{7}$.

O presente estudo tem por objetivo apontar os sinais, sintomas e complicações neurológicas relatados em pacientes infectados pelo SARS-CoV-2.

\section{MÉTODO}

O estudo consiste em uma revisão da literatura, na qual foram incluídos artigos científicos publicados em revistas indexadas, que traziam como tema principal o acometimento neurológico pelo SARS-CoV-2, mediante a aplicação do processo metodológico de identificar, triar, eleger e incluir.

A pesquisa foi realizada nas bases de dados PUBMED, EMBASE e Google Acadêmico sem restrição de idiomas. Houve a combinação das palavras-chave "covid-19", "neurology" e "SARS-CoV-2", concomitante a aplicação de um filtro temporal restringindo os trabalhos produzidos no ano de 2020 e que foram publicados até o dia 30 de maio.

\section{RESULTADOS}

As buscas na literatura resultaram na inclusão de 13 artigos que reportavam associação do Sars-CoV-2 com o sistema nervoso por meio de sinais, sintomas e complicações neurológicas. Esses artigos continham o relato de caso de 27 
pacientes no total. O sintoma mais comum relatado foi febre, presente em $48,14 \%$ dos pacientes, seguido por paresias em $40,74 \%$ e estado mental alterado em 33,33\% dos indivíduos. Distúrbios do olfato e do paladar estão presentes em $14,81 \%$ dos casos. Afasia foi descrita em 7,40\% dos pacientes, disartria em 25,92\%, ataxia $14,81 \%$ e hemiplegia em $22,22 \%$ dos casos.

Outros sintomas relevantes presentes foram: hemianopsia homônima $(7,4 \%)$, dor de cabeça $(7,4 \%)$, rigidez nucal $(7,4 \%)$, inapetência $(7,4 \%)$, convulsão $(7,4 \%)$, alodinia distal $(3,7 \%)$ e síncope $(3,7 \%)$. A média de idade de pacientes em que o SARS-CoV-2 gerou sintomas neurológicos foi de 55,6 anos. O paciente mais velho desse estudo foi descrito por Avula e colaboradores (2020) e tinha 88 anos, e evoluiu com $\mathrm{AVC}^{8}$, enquanto o paciente mais jovem possui 24 anos de idade, teve encefalite e foi descrito por Moriguchi et al (2020) ${ }^{9}$.

Quanto aos acometimentos neurológicos relatados na literatura em pacientes infectados com o SARS-CoV-2 que tiveram repercussões neurológicas, 48,14\% sofreram algum tipo de acidente vascular cerebral (AVC), enquanto 25,92\% cursaram com Síndrome de Guillain Barré (SGB) e 14,81\% evoluíram com encefalite. Muhammad e colaboradores (2020), relataram um caso de aneurisma e hemorragia subaracnóidea ${ }^{10}$. 
Quadro 1. Mostra os principais achados neurológicos de pacientes com SARS-CoV-2 por artigo analisado neste estudo.

\begin{tabular}{|c|c|c|c|c|c|c|}
\hline Artigo & $\begin{array}{l}\text { No de } \\
\text { casos }\end{array}$ & Caso & $\begin{array}{l}\text { Sinais e } \\
\text { sintomas } \\
\text { neurológicos }\end{array}$ & $\begin{array}{l}\text { Complicação } \\
\text { Neurológica }\end{array}$ & $\begin{array}{l}\text { Idade } \\
\text { (anos) }\end{array}$ & Referência \\
\hline $\begin{array}{l}\text { Encephalitis as a } \\
\text { clinical } \\
\text { manifestation of } \\
\text { COVID-19 }\end{array}$ & 01 & 01 & $\begin{array}{l}\text { Febre, mialgia, } \\
\text { estado mental } \\
\text { alterado, rigidez } \\
\text { nucal, sinal de } \\
\text { Kernig e } \\
\text { Brudzinski } \\
\text { positivos }\end{array}$ & Encefalite & - & Ye $2020^{11}$ \\
\hline \multirow[t]{4}{*}{$\begin{array}{l}\text { COVID-19 } \\
\text { presenting as } \\
\text { stroke }\end{array}$} & 04 & 01 & $\begin{array}{l}\text { Febre, estado } \\
\text { mental alterado, } \\
\text { vômito e } \\
\text { ingestão oral } \\
\text { reduzida }\end{array}$ & AVC & 73 & Avula $2020^{8}$ \\
\hline & & 02 & $\begin{array}{l}\text { Febre, } \\
\text { hemiparesia } \\
\text { esquerda, } \\
\text { disartria, } \\
\text { inapetência }\end{array}$ & AVC & 83 & \\
\hline & & 03 & $\begin{array}{l}\text { Estado mental } \\
\text { alterado, } \\
\text { hemiplegia } \\
\text { esquerda e } \\
\text { afasia }\end{array}$ & AVC & 80 & \\
\hline & & 04 & $\begin{array}{l}\text { Fraqueza e } \\
\text { dormência no } \\
\text { braço direito e } \\
\text { afasia }\end{array}$ & AVC & 88 & \\
\hline $\begin{array}{l}\text { Severe brain } \\
\text { haemorrhage and } \\
\text { concomitant } \\
\text { COVID-19 } \\
\text { Infection: A } \\
\text { neurovascular } \\
\text { complication of } \\
\text { COVID-19 }\end{array}$ & 01 & 01 & $\begin{array}{l}\text { Estado mental } \\
\text { alterado }\end{array}$ & $\begin{array}{c}\text { Hemorragia } \\
\text { subaracnóidea, } \\
\text { aneurisma } \\
\text { intracraniano }\end{array}$ & 60 & $\begin{array}{l}\text { Muhammad } \\
2020^{10}\end{array}$ \\
\hline $\begin{array}{l}\text { A Case of COVID- } \\
19 \text { Encephalitis }\end{array}$ & 01 & 01 & Febre e síncope & Encefalite & 40 & Huang $2020^{12}$ \\
\hline $\begin{array}{l}\text { Guillain Barre } \\
\text { syndrome } \\
\text { associated with } \\
\text { COVID-19 } \\
\text { infection: A case } \\
\text { report }\end{array}$ & 01 & 01 & $\begin{array}{l}\text { Febre, } \\
\text { tetraparesia e } \\
\text { paresia facial } \\
\text { bilateral }\end{array}$ & SGB & 65 & Sedaghat $2020^{13}$ \\
\hline $\begin{array}{l}\text { Guillain-Barré } \\
\text { Syndrome as a } \\
\text { Complication of } \\
\text { SARS-CoV-2 } \\
\text { Infection }\end{array}$ & 01 & 01 & $\begin{array}{l}\text { Paraparesia, } \\
\text { alodinia distal e } \\
\text { constipação }\end{array}$ & SGB & 70 & Coen $2020^{14}$ \\
\hline
\end{tabular}


Quadro 1 (cont.). Mostra os principais achados neurológicos de pacientes com SARS-CoV-2 por artigo analisado neste estudo.

\begin{tabular}{|c|c|c|c|c|c|c|}
\hline \multirow[t]{4}{*}{$\begin{array}{l}\text { Coexistence of } \\
\text { Covid-19 and Acute } \\
\text { Ischemic Stroke } \\
\text { Report of Four } \\
\text { Cases }\end{array}$} & 04 & 01 & $\begin{array}{l}\text { Paresia facial } \\
\text { esquerda, } \\
\text { disartria, } \\
\text { hemiparesia } \\
\text { esquerda e } \\
\text { febre }\end{array}$ & AVC & 45 & Tunç $2020^{15}$ \\
\hline & & 02 & $\begin{array}{l}\text { Disartria, } \\
\text { hemiparesia } \\
\text { direita e febre }\end{array}$ & AVC & 67 & \\
\hline & & 03 & $\begin{array}{l}\text { Estado mental } \\
\text { alterado, } \\
\text { disartria e } \\
\text { hemiparesia } \\
\text { direita }\end{array}$ & AVC & 72 & \\
\hline & & 04 & $\begin{array}{l}\text { Febre, hemi- } \\
\text { hipoestesia e } \\
\text { ataxia }\end{array}$ & AVC & 77 & \\
\hline \multirow[t]{5}{*}{$\begin{array}{l}\text { Large-Vessel } \\
\text { Stroke as a } \\
\text { Presenting Feature } \\
\text { of Covid-19 in the } \\
\text { Young }\end{array}$} & 05 & 01 & $\begin{array}{l}\text { Hemiparesia, } \\
\text { hemiplegia do } \\
\text { lado esquerdo, } \\
\text { hemianopsia } \\
\text { homônima, } \\
\text { disartria e } \\
\text { déficit sensorial }\end{array}$ & AVC & 33 & Oxley $2020^{16}$ \\
\hline & & 02 & $\begin{array}{l}\text { Estado mental } \\
\text { alterado, } \\
\text { disfasia, } \\
\text { hemiplegia no } \\
\text { lado direito, } \\
\text { disartria e } \\
\text { déficit sensorial }\end{array}$ & AVC & 37 & \\
\hline & & 03 & $\begin{array}{l}\text { Estado mental } \\
\text { alterado, } \\
\text { hemianopsia } \\
\text { homônima } \\
\text { esquerda, } \\
\text { hemiplegia no } \\
\text { lado esquerdo e } \\
\text { ataxia }\end{array}$ & AVC & 39 & \\
\hline & & 04 & $\begin{array}{l}\text { Estado mental } \\
\text { alterado, } \\
\text { disfasia e } \\
\text { hemiplegia no } \\
\text { lado direito }\end{array}$ & AVC & 44 & \\
\hline & & 05 & $\begin{array}{l}\text { Estado mental } \\
\text { alterado, } \\
\text { hemiplegia à } \\
\text { esquerda e } \\
\text { disartria }\end{array}$ & AVC & 49 & \\
\hline
\end{tabular}

SGB - Síndrome de Guillain Barré. AVC - Acidende Vascular Cerebral 
Quadro 1 (cont.). Mostra os principais achados neurológicos de pacientes com SARS-CoV-2 por artigo analisado neste estudo.

\begin{tabular}{|c|c|c|c|c|c|c|}
\hline \multirow[t]{5}{*}{$\begin{array}{l}\text { Guillain-Barré } \\
\text { Syndrome } \\
\text { Associated with } \\
\text { SARS-CoV-2 }\end{array}$} & 05 & 01 & $\begin{array}{l}\text { Febre, ageusia, } \\
\text { quadriplegia e } \\
\text { parestesia dos } \\
\text { membros } \\
\text { superiores }\end{array}$ & SGB & - & Toscano $2020^{17}$ \\
\hline & & 02 & $\begin{array}{l}\text { Febre, diplegia } \\
\text { facial, } \\
\text { Tetraplegia } \\
\text { generalizada, } \\
\text { areflexia, } \\
\text { parestesia e } \\
\text { ataxia }\end{array}$ & SGB & - & \\
\hline & & 03 & $\begin{array}{l}\text { Febre,tetrapare } \\
\text { siae areflexia }\end{array}$ & SGB & - & \\
\hline & & 04 & $\begin{array}{l}\text { Hiposmia, } \\
\text { tetraparesia e } \\
\text { ataxia }\end{array}$ & SGB & - & \\
\hline & & 05 & $\begin{array}{l}\text { Ageusia, } \\
\text { anosmia e } \\
\text { paraplegia }\end{array}$ & SGB & - & \\
\hline $\begin{array}{l}\text { Paciente con clínica } \\
\text { neurológica como } \\
\text { única } \\
\text { manifestación de } \\
\text { infección por } \\
\text { SARS-CoV-2 }\end{array}$ & 01 & 01 & $\begin{array}{l}\text { Tontura, } \\
\text { anosmia e } \\
\text { ageusia }\end{array}$ & - & - & Pellitero $2020^{18}$ \\
\hline $\begin{array}{l}\text { A first case of } \\
\text { meningitis/encepha } \\
\text { litis associated with } \\
\text { SARS-Coronavirus- } \\
2\end{array}$ & 01 & 01 & $\begin{array}{l}\text { Dor de cabeça, } \\
\text { febre, } \\
\text { convulsões e } \\
\text { rigidez nucal }\end{array}$ & Encefalite & 24 & Moriguchi $2020^{9}$ \\
\hline $\begin{array}{l}\text { Magnetic } \\
\text { Resonance Imaging } \\
\text { Alteration of the } \\
\text { Brain in a Patient } \\
\text { With Coronavirus } \\
\text { Disease } 2019 \\
\text { (COVID-19) and } \\
\text { Anosmia }\end{array}$ & 01 & 01 & $\begin{array}{l}\text { Anosmia e } \\
\text { disgeusia }\end{array}$ & - & 25 & Politi $2020^{19}$ \\
\hline $\begin{array}{l}\text { Meningoencephaliti } \\
\text { s without } \\
\text { respiratory failure } \\
\text { in a young female } \\
\text { patient with } \\
\text { COVID-19 infection } \\
\text { in Downtown Los } \\
\text { Angeles, early April } \\
2020\end{array}$ & 01 & 01 & $\begin{array}{l}\text { Dor de cabeça, } \\
\text { febre e } \\
\text { convulsão }\end{array}$ & Encefalite & 41 & Duong $2020^{20}$ \\
\hline
\end{tabular}

SGB - Síndrome de Guillain Barré. AVC - Acidende Vascular Cerebral 


\section{DISCUSSÃO}

\section{Mecanismos de invasão do SNC pelo SARS-CoV-2}

Embora já se tenha encontrado o SARS-CoV-2 no líquido cerebrospinal e tecido encefálico de pacientes com a COVID-194,7, não se tem até o momento a elucidação de como ocorre a invasão dos tecidos nervosos pelo vírus. Acredita-se que possa ocorrer por via transináptica em células nervosas infectadas, via bulbo olfatório, endotélio vascular infectado ou por leucócitos que migram através da barreira hematoencefálica $(\mathrm{BHE})^{6}$.

A enzima conversora de angiotensina 2 (ECA2) foi identificada como o receptor do SARS-CoV-2 no organismo, esta interage a partir da proteína spike (S), por meio do domínio de ligação da proteína S. Células nervosas possuem ECA2 e, portanto, são passíveis de infecção pelo vírus. A ligação do SARS-CoV-2 aos receptores ECA2 na membrana celular é mediada pela proteína spike $1(\mathrm{~S} 1)^{2,3,7}$. O SARSCoV-2, possui subunidades S1 e S2, com função de interagir e efetuar a ligação em receptores celulares. O resíduo lisina 31 do receptor ECA2 reconhece com muita afinidade o sítio ativo de ligação ao receptor, que possui um resíduo 394 da glutamina. Com a interação uma mudança espacial na proteína spike ocorre e a invasão é concretizada. A ligação de S1 com ECA2 é primordial, mas o domínio S2 é o encarregado da fusão à membrana celular e invasão viral, com consequente ativação de proteases celulares como a transmembranar serina 2 (TMPRSS2). No meio intracelular, após um processo de tradução de proteínas, ocorre quebra 
destas para a formação de peptídeos não estruturais. Por fim, a montagem dos novos vírus é efetuada pelo Complexo de Golgi e retículo endoplasmático, com formação de vesículas e exocitose ${ }^{3,21-24}$.

Uma das hipóteses prováveis da disseminação para tecidos secundários, como o nervoso, é a propagação de forma retrógrada, que consiste na infecção via transináptica através do bulbo olfatório. É baseada na ideia de invasão de nervos periféricos com deslocamento do vírus até alcançar o sistema nervoso central (SNC). Nesse caso, acredita-se que o vírus se liga a estrutura de transporte dos neurônios, os microtúbulos, e seja secretado no espaço sináptico por intermédio da formação de vesículas. No caso do bulbo olfatório, acredita-se que a invasão ao SNC ocorre pela placa cribriforme do osso etmoide $e^{6,21,25}$. Outra hipótese seria a invasão de estruturas bulbares relacionadas à respiração como os núcleos ambíguo e do trato solitário ${ }^{21}$. Estudos recentes, sugerem que células tronco olfativas são portadoras da ECA2, enquanto terminações nervosas da cavidade nasal não, o que significa que não há invasão através de nervos olfatórios e a anosmia é consequência da invasão das células portadoras da ECA2 ${ }^{5}$.

Outra forma sugerida de disseminação é via sanguínea, na qual leucócitos e endotélio vascular são infectados. Células endoteliais em vários tecidos do organismo expressam a ECA2, e autópsias de indivíduos mortos pela COVID-19 evidenciam endocitose e exocitose de partículas virais no SNC por células epiteliais ${ }^{6,25}$. Os linfócitos T podem 
ser infectados pelo SARS-CoV-2. Nesse contexto, a inflamação sistêmica causada pela COVID-19 causa um aumento de permeabilidade da $\mathrm{BHE}$, o que permite que células $T$ infectadas penetrem-na em um processo denominado Cavalo de Troia ${ }^{6}$. No SNC, monócitos infectados causam neuroinflamação por meio da liberação de citocinas pró-inflamatórias com posterior ativação e recrutamento microglial ao sítio de inflamação 5,6,25.

\section{Manifestações neurológicas da COVID-19}

Estudos com pacientes falecidos em decorrência da COVID-19 apontam degeneração neuronal e edema cerebral. A dor de cabeça é um dos sintomas relatados pelos infectados pela COVID-1921. A relação fisiopatológica existente entre a COVID-19 e a dor de cabeça ainda não está clara, mas acredita-se que com a neuroinflamação, mecanismos da resposta imune são desencadeados, resultando em liberação de citocinas e quimiocinas, que ativam neurônios sensitivos nociceptivos ${ }^{6,21}$.

Distúrbios do olfato e paladar se apresentam comuns com relatos de que a anosmia antecede a ageusia21,25. A encefalopatia cursa com disfunção cerebral transitória e manifestação por meio de confusão mental, delírio, letargia ou coma6. Quando se trata de COVID-19, esse acontecimento é mais frequente em idosos, portadores de comorbidades, desnutridos, presença de infecção e de riscos vasculares $^{5,7}$. Distúrbios metabólicos e endócrinos que evoluem com baixas e altas concentrações de sódio, cálcio 
ou glicose, disfunções relacionadas a rins e fígado, sepse e a tempestade inflamatória de citocinas aumentam a possibilidade de encefalopatia. Além disso, SARS-CoV-2 proporciona ao paciente episódios de hipoxemia grave, que pode levar a um maior risco de desenvolvimento de encefalopatia6,21. Encefalite foi relatada em paciente que possuía o SARS-CoV-2 no líquido cerebrospinal e que não tinha carga viral em material biológico da nasofaringe. Concentrações séricas altas no líquido cerebrospinal de citocinas inflamatórias podem aumentar a permeabilidade da BHE e permitir a entrada do vírus no SNC ${ }^{6,21,25}$.

O nível de consciência debilitado já está sendo bem documentado e algumas hipóteses de sua origem seria uma lesão direta ao parênquima do tecido nervoso, encefalopatias, desenvolvimento de doenças desmielinizantes e convulsões ${ }^{2,6}$.

No tocante ao AVC, acredita-se que a fisiopatologia é multifatorial, mas se sabe que a proteína spike interage com receptores ECA2 e que isso pode promover aumento da pressão arterial, que combinado com trombocitopenia, lesão endotelial e distúrbios hematogênicos podem culminar no desencadeamento de AVC isquêmico e hemorrágico ${ }^{6}$. Outro fator que pode contribuir para a ocorrência de acidentes cerebrovasculares é a síndrome da tempestade de citocinas. Achados laboratoriais comuns em pacientes que evoluíram com AVC concomitante a infecção pelo SARS-CoV-2 é número alto de leucócitos, proteína C reativa, lactato, dímero-D e ferritina ${ }^{21}$. Outra complicação neurológica que 
vêm sendo documentada na literatura é SGB, em que se acredita que por meio de um mimetismo celular o vírus portador de epítopos muito semelhantes a epítopos de nervos, sensibilizam células imunes autorreativas, resultando na produção de auto anticorpos e provocam lesão neuronal 6 .

\section{CONCLUSÃO}

Ainda há poucas evidências que permitam afirmar com precisão quais são os mecanismos exatos de invasão do tecido nervoso, mas já se tem a evidência que esse processo existe. Relatos da concomitância da infecção por COVID-19 com acometimentos neurológicos podem indicar o possível neurotropismo desse vírus.

Ainda é cedo para saber se esse vírus tem capacidade de causar acometimentos a longo prazo por meio de lesão neuronal, o que só será possível afirmar mediante estudos prospectivos futuros acompanhando esses pacientes. Até agora o que se sabe é que a doença está implicada em sintomas e acometimentos neurológicos nos pacientes infectados. Mais estudos são necessários para se esclarecer quais fatores podem contribuir para o acometimento neurológico, além de quais fatores poderiam representar efeito neuroprotetor para os pacientes infectados pelo SARSCoV-2. 


\section{REFERÊNCIAS}

1. Guan W, Ni Z, Hu Y, Liang W, Ou C, He J, et al. Clinical Characteristics of Coronavirus Disease 2019 in China. N Engl J Med 2020;382:170820. https://doi.org/10.1056/NEJMoa2002032

2.Baig AM, Khaleeq A, Ali U, Syeda H. Evidence of the COVID-19 Virus Targeting the CNS: Tissue Distribution, Host-Virus Interaction, and Proposed Neurotropic Mechanisms. ACS Chem Neurosci 2020;11:9958. https://doi.org/10.1021/acschemneuro.0c00122

3.Lan J, Ge J, Yu J, Shan S, Zhou H, Fan S, et al. Structure of the SARS-CoV-2 spike receptor- binding domain bound to the ACE2 receptor. Nature 2020;518:215-20. https://doi.org/10.1038/s41586020-2180-5

4.Asadi-Pooya AA, Simani L. Central nervous system manifestations of COVID-19: A systematic review. J Neurol Sci 2020;413:1-4. https://doi.org/10.1016/j.jns.2020.116832

5. Kwong KCNK, Mehta PR, Skukla G, Mehta AR. COVID-19, SARS and MERS: A neurological perspective. J Clin Neurosci 2020;77:13-6. https://doi.org/10.1016/j.jocn.2020.04.124

6.Zubair AS, McAlpine LS, Gardin T, Farhadian S, Kuruvilla DE, Spudich S. Neuropathogenesis and Neurologic Manifestations of the Coronaviruses in the Age of Coronavirus Disease 2019 A Review. JAMA Neurol 2020;77:1018-27. https://doi.org/10.1001/jamaneurol.2020.2065

7. Mao L, Jin H, Wang M, Hu Y, Chen S, He Q, et al. Neurologic Manifestations of Hospitalized Patients With Coronavirus Disease 2019 inWuhan, China. JAMA Neurol 2020;77:683-90. https://doi.org/10.1001/jamaneurol.2020.1127

8. Avula A, Nalleballe K, Narula N, Sapozhnikov S, Dandu V, Toom S, et al. COVID-19 presenting as stroke. Brain Behav Immun 2020;87:115-9. https://doi.org/10.1016/j.bbi.2020.04.077

9. Moriguchi T, Harii N, Goto J, Harada D, Sugawara H, Takamino J, et al. A first case of meningitis/encephalitis associated with SARSCoronavirus-2. Int J Infect Dis 2020;94:55-8. https://doi.org/10.1016/j.ijid.2020.03.062

10. Muhammad S, Petridis A, Cornelius JF, Hänggi D. Severe brain haemorrhage and concomitant COVID-19 Infection: A neurovascular complication of COVID-19. Brain Behav Immun 2020;87:150-1. https://doi.org/10.1016/j.bbi.2020.05.015

11. Ye M, Ren Y, LV T. Encephalitis as a clinical manifestation of COVID19. Brain Behav Immun 2020;88:945-6. https://doi.org/10.1016/j.bbi.2020.04.017

12. Huang $\mathrm{H}$, Jiang $\mathrm{D}$, Huang JT. SARS-CoV-2 Detected in Cerebrospinal Fluid by PCR in a Case of COVID-19 Encephalitis. Brain Behav Immun 2020;87:149. https://doi.org/10.1016/j.bbi.2020.05.012

13.Sedaghat Z, Karimi N. Guillain Barre syndrome associated with COVID-19 infection: A case report. J Clin Neurosci 2020;76:233-5. https://doi.org/10.1016/j.jocn.2020.04.062

14.Coen M, Jeanson G, Almeida LAC, Hübers A, Stierlin F, Najjar I, et al. Guillain-Barré Syndrome as a Complication of SARS-CoV-2 
Infection. Brain Behav Immun 2020;87:111-2. https://doi.org/10.1016/j.bbi.2020.04.074

15.Tunç A, Ünlübaş Y, Alemdar M, Akyüz E. Coexistence of Covid-19 and Acute Ischemic Stroke Report of Four Cases. J Clin Neurosci 2020;77:227-9. https://doi.org/10.1016/j.jocn.2020.05.018 16. Oxley TJ, Mocco J, Majidi S, Kellner CP, Shoirah H, Singh P, et al. Large-Vessel Stroke as a Presenting Feature of Covid-19 in the Young. N Engl J Med 2020;382:e60. https://doi.org/10.1056/NEJMc2009787 17.Toscano $G$, Palmerini $F$, Ravaglia $S$, Ruiz $L$, Invernizzi $P$, Cuzzoni MG, et al. Guillain-Barré Syndrome Associated with SARS-CoV-2. N Engl J Med 2020;382:2574-6. https://doi.org/10.1056/NEJMc2009191 18.Pellitero SE, Ferrer-Bergua L. Paciente con clínica neurológica como única manifestación de infección por SARS-CoV-2. Neurologia 2020;35:271-2. https://doi.org/10.1016/j.nrl.2020.04.010

19.Politi LS, Salsano E, Grimaldi M. Magnetic Resonance Imaging Alteration of the Brain in a Patient With Coronavirus Disease 2019 (COVID-19) and Anosmia. JAMA Neurol 2020;77:1028-9. https://doi.org/10.1001/jamaneurol.2020.2125

20.Duong L, Xu P, Liu A. Meningoencephalitis without respiratory failure in a young female patient with COVID-19 infection in Downtown Los Angeles, early April 2020. Brain Behav Immun 2020;87:33. https://doi.org/10.1016/j.bbi.2020.04.024

21. Carod-Artal FJ. Complicaciones neurológicas por coronavirus y COVID-19. Rev Neurol 2020;70:311-22. https://doi.org/10.33588/rn.7009.2020179

22. Harapan $\mathrm{H}$, Itoh $\mathrm{N}$, Yufika A, Winardi $\mathrm{W}$, Keam $\mathrm{S}, \mathrm{Te} \mathrm{H}$, et al. Coronavirus disease 2019 (COVID-19): A literature review. J Infect Public Health 2020;13:667-73. https://doi.org/10.1016/j.jiph.2020.03.019

23.Shang J, Ye G, Shi K, Wan Y, Luo C, Aihara H, et al. Structural basis of receptor recognition by SARS-CoV-2. Nature 2020;581:221-38. https://doi.org/10.1038/s41586-020-2179-y

24.Sungnak W, Huang N, Bécavin C, Berg M, Queen R, Litvinukova M, et al. SARS-CoV-2 entry factors are highly expressed in nasal epithelial cells together with innate immune genes. Nat Med 2020;26:681-7. https://doi.org/10.1038/s41591-020-0868-6

25.Troyer EA, Kohn JN, Hong S. Are we facing a crashing wave of neuropsychiatric sequelae of COVID-19? Neuropsychiatric symptoms and potential immunologic mechanisms. Brain Behav Immun 2020;87:34-9. https://doi.org/10.1016/j.bbi.2020.04.027 